YITP-SB-09-28

\title{
Hidden Sector Models and Signatures
}

\author{
Zuowei Liu ${ }^{\text {a }}$ \\ ${ }^{a}$ C.N. Yang Institute for Theoretical Physics, Stony Brook University, \\ Stony Brook, New York 11794-3840, USA
}

In the Stueckelberg extension of the Standard Model (StSM), matter in the hidden sector can act as dark matter. Due to an interplay of mixings produced by the usual Higgs mechanism and the Stueckelberg mechanism in the neutral gauge boson sector, the hidden sector matter acquires a milli charge. The Stueckelberg extension also produces a narrow width $\mathrm{Z}$ prime which is detectable at the Large Hadron Collider. The hidden sector dark matter naturally explains the PAMELA positron excess by means of a Breit-Wigner enhancement through a $\mathrm{Z}$ prime resonance. We also discuss the origin of milli charge in the context of the kinetic mixing and the Stueckelberg mixing.

\section{Introduction}

A Stueckelberg mechanism allows an Abelian gauge boson to develop a mass without the benefit of a Higgs mechanism (For the early history of the Stueckelberg mechanism see, [1|2|3] and [45]). The Stueckelberg Lagrangian couples one Abelian gauge boson $A_{\mu}$ to one pseudo-scalar $\sigma$ in the following way

$\mathcal{L}=-\frac{1}{4} F_{\mu \nu} F^{\mu \nu}-\frac{1}{2}\left(m A_{\mu}+\partial_{\mu} \sigma\right)\left(m A^{\mu}+\partial^{\mu} \sigma\right)(1)$

which is gauge invariant under the transformation

$$
\begin{aligned}
\delta A_{\mu} & =\partial_{\mu} \lambda, \\
\delta \sigma & =-m \lambda .
\end{aligned}
$$

The gauge boson mass growth through the Stueckelberg couplings can occur naturally in compactifications of higher-dimensional string theory, supergravity, or even pure gauge theory. Here we review the Stueckelberg extension of the Standard Model. (For Stueckelberg extensions of the SUSY models, see [67/8 9 10 11 12 13].) We only review the Stueckelberg models where the extra $U(1)$ is not anomalous. (For the anomalous $U(1)$ case, see [14]15 16 17|18, 1920 21 22].)

\section{Stueckelberg Extension of the Standard Model (StSM)}

The Stueckelberg Lagrangian can extend the Standard Model to include additional $U(1)$ gauge fields [23]. The simplest case is to extend the Standard
Model gauge group to have one additional $U(1)$, i.e., $S U(3)_{C} \times S U(2)_{L} \times U(1)_{Y} \times U(1)_{X}$. Thus in this case the total Lagrangian reads

$$
\begin{aligned}
\mathcal{L}_{\mathrm{StSM}}= & \mathcal{L}_{\mathrm{SM}}+\mathcal{L}_{\mathrm{St}} \\
\mathcal{L}_{\mathrm{St}}= & -\frac{1}{4} C_{\mu \nu} C^{\mu \nu}+g_{X} C_{\mu} J_{X}^{\mu} \\
& -\frac{1}{2}\left(\partial_{\mu} \sigma+M_{1} C_{\mu}+M_{2} B_{\mu}\right)^{2}
\end{aligned}
$$

where $C_{\mu}$ is the gauge field associated with $U(1)_{X}$ and $B_{\mu}$ is the SM hypercharge gauge field; $g_{X}$ is the gauge coupling strength of the $U(1)_{X}$ and $J_{X}^{\mu}$ is the conserved fermion matter current that the gauge filed $C_{\mu}$ couples to. In general, the interaction between the gauge field $C_{\mu}$ and matter fields can include both the Standard Model fermion sectors (visible sector) and hidden fermion sector matter fields. However, the interaction between the $U(1)_{X}$ gauge boson and the visible sector matter can lead to modifications of the charge of the neutron which is measured to a high precision [8]. To avoid this potential violation, we assume that the Standard Model fields are neutral under $U(1)_{X}$. It is easy to check that the above Lagrangian is invariant under the $U(1)_{X} \times U(1)_{Y}$ transformation

$\delta_{X}\left(C_{\mu}, B_{\mu}, \sigma\right)=\left(\partial_{\mu} \lambda_{X}, 0,-M_{1} \lambda_{X}\right)$

$\delta_{Y}\left(C_{\mu}, B_{\mu}, \sigma\right)=\left(0, \partial_{\mu} \lambda_{Y},-M_{2} \lambda_{Y}\right)$.

The addition of the new gauge boson $C_{\mu}$ enlarges the vector boson neutral sector. In the basis $V_{\mu}^{T}=$ $\left(C_{\mu}, B_{\mu}, A_{\mu}^{3}\right)$, the mass matrix then takes the follow- 
ing form

$M^{2}=\left[\begin{array}{ccc}M_{1}^{2} & M_{1}^{2} \epsilon & 0 \\ M_{1}^{2} \epsilon & M_{1}^{2} \epsilon^{2}+\frac{1}{4} v^{2} g_{Y}^{2} & -\frac{1}{4} v^{2} g_{2} g_{Y} \\ 0 & -\frac{1}{4} v^{2} g_{2} g_{Y} & \frac{1}{4} v^{2} g_{2}^{2}\end{array}\right]$,

where $\epsilon \equiv M_{2} / M_{1} ; g_{2}$ and $g_{Y}$ are the $S U(2)_{L}$ and $U(1)_{Y}$ gauge coupling constants, and are normalized so that $M_{W}^{2}=g_{2}^{2} v^{2} / 4$. This allows one to choose $\epsilon$ and $M_{1}$ as two independent parameters to characterize physics beyond the SM. It is easily checked that $\operatorname{det}\left(M^{2}\right)=0$ which implies that one of the eigenvalues is zero, whose eigenvector we identify with the photon. The remaining two eigenvalues are nonvanishing and correspond to the $Z$ and $Z^{\prime}$ bosons.

The symmetric matrix $M^{2}$ can be diagonalized by an orthogonal transformation, $V=\mathcal{O} E$, with $E_{\mu}^{T}=$ $\left(Z_{\mu}^{\prime}, Z_{\mu}, A_{\mu}^{\gamma}\right)$. The transformation matrix $\mathcal{O}$ can be parameterized by three angles as follows

$\mathcal{O}=\left[\begin{array}{ccc}c_{\psi} c_{\phi}-s_{\theta} s_{\phi} s_{\psi} & s_{\psi} c_{\phi}+s_{\theta} s_{\phi} c_{\psi} & -c_{\theta} s_{\phi} \\ c_{\psi} s_{\phi}+s_{\theta} c_{\phi} s_{\psi} & s_{\psi} s_{\phi}-s_{\theta} c_{\phi} c_{\psi} & c_{\theta} c_{\phi} \\ -c_{\theta} s_{\psi} & c_{\theta} c_{\psi} & s_{\theta}\end{array}\right]$

where $s_{\theta}=\sin \theta, c_{\theta}=\cos \theta$, etc. The angels are defined as [8]

$\tan \phi=\epsilon$,

$\tan \theta=\frac{g_{Y}}{g_{2} \sqrt{1+\epsilon^{2}}}$,

$\tan \psi=\frac{\tan \theta \tan \phi M_{W}^{2}}{\cos \theta\left(M_{Z^{\prime}}^{2}-M_{W}^{2}\left(1+\tan ^{2} \theta\right)\right)}$.

There is also a modification of the expression of the electric charge in terms of the fundamental physics couplings. Thus if we write the EM interaction in the form $e A_{\mu}^{\gamma} J_{\mathrm{em}}^{\mu}$ the expression for $e$ is given by

$\frac{1}{e^{2}}=\frac{1}{g_{2}^{2}}+\frac{1+\epsilon^{2}}{g_{Y}^{2}}$.

Thus $g_{Y}$ is related to $g_{Y}^{\mathrm{SM}}$ by

$g_{Y}=g_{Y}^{\mathrm{SM}} \sqrt{1+\epsilon^{2}}$.

In the limit $\epsilon \rightarrow 0$, when the Abelian $C_{\mu}$ field decouples from SM, the mixing angles $\phi$ and $\psi$ vanish, and the hypercharge coupling $g_{Y}$ recovers its SM value.

\section{Stueckelberg Extension of the Standard Model with Kinetic Mixing (StkSM)}

We discuss here the StSM model in the context of kinetic mixing between $U(1)_{X}$ and $U(1)_{Y}$. Such kinetic mixing is quite generic, and can arise in a variety of ways in a broad class of models. We will characterize the kinetic mixing term as follows

$\mathcal{L}_{\mathrm{KM}}=-\frac{\delta}{2} C_{\mu \nu} B^{\mu \nu}$,

and the total Lagrangian of StkSM is $\mathcal{L}_{\mathrm{StkSM}}=$ $\mathcal{L}_{\mathrm{StSM}}+\mathcal{L}_{\mathrm{KM}}$ where $\mathcal{L}_{\mathrm{StSM}}$ is given in Eq.(4). Thus, the model consists of both the kinetic mixing and the Stueckelberg mass mixing [2425]. The kinetic mixing Lagrangian leads to non-diagonal elements in the kinetic energy Lagrangian in the basis $V_{\mu}^{T}=$ $\left(C_{\mu}, B_{\mu}, A_{\mu}^{3}\right)$

$\mathcal{K}=\left[\begin{array}{lll}1 & \delta & 0 \\ \delta & 1 & 0 \\ 0 & 0 & 1\end{array}\right]$

A simultaneous diagonalization of both the kinetic energy terms, Eq.(15), and the mass matrix, Eq.(7) can be achieved by a transformation $T=K R$, which is a combination of a $G L(3)$ transformation $(K)$ and an orthogonal transformation $(R)$. This allows one to work in the mass basis with the transformation $E_{\mu}=R^{T} K^{T} V_{\mu}$. A special choice of the $G L(3)$ transformation $(K)$ as follows

$K=\left[\begin{array}{ccc}1 & -\delta / \sqrt{1-\delta^{2}} & 0 \\ 0 & 1 / \sqrt{1-\delta^{2}} & 0 \\ 0 & 0 & 1\end{array}\right]$

diagonalizes the kinetic energy terms, and at the same time transforms the mass matrix, Eq.(7) into

$M^{2}=\left[\begin{array}{ccc}M_{1}^{2} & M_{1}^{2} \bar{\epsilon} & 0 \\ M_{1}^{2} \bar{\epsilon} & M_{1}^{2} \bar{\epsilon}^{2}+\frac{1}{4} v^{2} \bar{g}_{Y}^{2} & -\frac{1}{4} v^{2} g_{2} \bar{g}_{Y} \\ 0 & -\frac{1}{4} v^{2} g_{2} \bar{g}_{Y} & \frac{1}{4} v^{2} g_{2}^{2}\end{array}\right]$

The above mass matrix in StkSM looks exactly the same as the one in StSM, Eq. (7), except that the parameters $\epsilon$ and $g_{Y}$ get redefined as follows

$\bar{\epsilon}=\frac{\epsilon-\delta}{\sqrt{1-\delta^{2}}}$,
$\bar{g}_{Y}=\frac{g_{Y}}{\sqrt{1-\delta^{2}}}$. 
Thus the orthogonal transformation $R\left(\bar{\epsilon}, \bar{g}_{Y}\right)$ has the same form as the orthogonal transformation $\mathcal{O}\left(\epsilon, g_{Y}\right)$ in StSM. The neutral sector interaction can now be written in the form

$\mathcal{L}_{\mathrm{NC}}=J^{\mu T} K(\delta) R\left(\bar{\epsilon}, \bar{g}_{Y}\right) E_{\mu}$

where $J^{\mu T}=\left(g_{X} J_{X}^{\mu}, g_{Y} J_{Y}^{\mu}, g_{2} J_{2}^{3 \mu}\right)$, so one finds that

$J^{\mu T} K(\delta)=\left[\begin{array}{c}g_{X} J_{X}^{\mu} \\ K_{12} g_{X} J_{X}^{\mu}+\bar{g}_{Y} J_{Y}^{\mu} \\ g_{2} J_{2}^{3 \mu}\end{array}\right]^{T}$

where $K_{12}=-\delta / \sqrt{1-\delta^{2}}$ is the matrix element of the $G L(3)$ transformation $(K)$, and we have used $\bar{g}_{Y}$ to absorb the matrix element $K_{22}$. If one restricts $J_{X}$ to only include the hidden sector matter, the interactions between Standard Model fermions and $E_{\mu}$ depend on $\bar{\epsilon}$ and $\bar{g}_{Y}$, not explicitly on $\delta$, and have the same form as in StSM. So one expects the same electric charge modification as in Eqs.(12, 13) in StkSM

$\bar{g}_{Y}=g_{Y}^{\mathrm{SM}} \sqrt{1+\bar{\epsilon}^{2}}$.

When substituting the modifications of the hypercharge coupling constants Eq.(13) and Eq.(22) back into the mass matrices Eq.(7) and Eq.(17), one finds that the mass matrices are identical except for the change of variable $\epsilon \rightarrow \bar{\epsilon}$. Thus the orthogonal transformation $R(\bar{\epsilon})$ depends only on $\bar{\epsilon}$, and has the form of the orthogonal transformation $\mathcal{O}(\epsilon)$ in StSM, i.e., $R=\mathcal{O}$. So one can conclude that the neutral sector interactions in StkSM are the same as in StSM, in the absence of the hidden sector matter. In order to discriminate the StkSM from StSM, one has to include the hidden sector.

The hidden sector matter is milli-charged with respect to the photon due to the small mixing between the two $U(1)$ fields. In StkSM, the milli charge $\left(Q_{\text {milli }}\right)$ of the hidden sector matter can be expressed as

$Q_{\text {milli }} \propto R_{13}(\bar{\epsilon})+K_{12}(\delta) R_{23}(\bar{\epsilon})$.

It is interesting to note that the milli charge $\left(Q_{\text {milli }}\right)$ vanishes when taking the limit $\epsilon \rightarrow 0$. Thus in Stueckelberg models, it is the Stueckelberg mass mixing $\epsilon$ that generates the milli charge of hidden sector matter, not the kinetic mixing $\delta$. We further discuss this topic in more details in the next section.

\section{Milli Charge in Stueckelberg models}

We illustrate the mechanism which generates the milli charge in Stueckelberg models. Consider a kinetic mixing model with two gauge fields $A_{1 \mu}, A_{2 \mu}$ corresponding to the gauge groups $U(1)$ and $U(1)^{\prime}$. We choose the following Lagrangian $\mathcal{L}=\mathcal{L}_{0}+\mathcal{L}_{1}$ where

$$
\begin{aligned}
& \mathcal{L}_{0}=-\frac{1}{4} F_{1 \mu \nu} F_{1}^{\mu \nu}-\frac{1}{4} F_{2 \mu \nu} F_{2}^{\mu \nu}-\frac{\delta}{2} F_{1 \mu \nu} F_{2}^{\mu \nu}, \\
& \mathcal{L}_{1}=J_{\mu}^{\prime} A_{1}^{\mu}+J_{\mu} A_{2}^{\mu} .
\end{aligned}
$$

Here $J_{\mu}$ is the physical source arising from quarks and leptons, and $J_{\mu}^{\prime}$ is the source from the hidden sector. To put the kinetic energy term in its canonical form, one may use the transformation

$K_{0}=\left[\begin{array}{cc}1 / \sqrt{1-\delta^{2}} & 0 \\ -\delta / \sqrt{1-\delta^{2}} & 1\end{array}\right]$.

However, the transformation is not unique, since $K=$ $K_{0} R$ instead of $K_{0}$ would do as well, where $R$ is an orthogonal matrix

$R=\left[\begin{array}{cc}\cos \theta & -\sin \theta \\ \sin \theta & \cos \theta\end{array}\right]$.

Thus $\mathcal{L}_{1}$ can be rewritten in terms of the new basis $A$ and $A^{\prime}$

$$
\begin{gathered}
\mathcal{L}_{1}=A^{\prime \mu}\left[\frac{\cos \theta}{\sqrt{1-\delta^{2}}} J_{\mu}^{\prime}+\left(\sin \theta-\frac{\cos \theta \delta}{\sqrt{1-\delta^{2}}}\right) J_{\mu}\right] \\
+A^{\mu}\left[-\frac{\sin \theta}{\sqrt{1-\delta^{2}}} J_{\mu}^{\prime}+\left(\cos \theta+\frac{\sin \theta \delta}{\sqrt{1-\delta^{2}}}\right) J_{\mu}\right] .
\end{gathered}
$$

In this case we see that both $A$ and $A^{\prime}$ can interact with $J$ and $J^{\prime}$. However, one may choose $\theta$ to get asymmetric solutions. For instance, one can take $\theta=\arctan \left[\delta / \sqrt{1-\delta^{2}}\right]$ to decouple $A^{\prime}$ from $J$. Here both $A$ and $A^{\prime}$ are massless.

Next we consider a model with kinetic mixing where a Stueckelberg mechanism generates a mass term of the type

$$
\begin{array}{r}
\mathcal{L}_{\text {Mass }}=-\frac{1}{2} M_{1}^{2} A_{1 \mu} A_{1}^{\mu}-\frac{1}{2} M_{2}^{2} A_{2 \mu} A_{2}^{\mu} \\
-M_{1} M_{2} A_{1 \mu} A_{2}^{\mu} .
\end{array}
$$

In this case diagonalizaton of the mass matrix fixes $\theta$ so that

$\theta=\arctan \left[\frac{\epsilon \sqrt{1-\delta^{2}}}{1-\delta \epsilon}\right]$, 
where $\epsilon \equiv M_{2} / M_{1}$ is the Stueckelberg mass mixing parameter. Thus the interaction Lagrangian in the new basis is given by

$$
\begin{array}{r}
\mathcal{L}_{1}=\frac{(\epsilon-\delta) J_{\mu}+(1-\delta \epsilon) J_{\mu}^{\prime}}{\sqrt{1-2 \delta \epsilon+\epsilon^{2}} \sqrt{1-\delta^{2}}} A_{M}^{\mu} \\
+\frac{J_{\mu}-\epsilon J_{\mu}^{\prime}}{\sqrt{1-2 \delta \epsilon+\epsilon^{2}}} A_{\gamma}^{\mu} .
\end{array}
$$

Here for the case $\epsilon=0$ one finds that the massless state, the photon $A_{\gamma}^{\mu}$, no longer couples with the hidden sector. We conclude, therefore, that in the absence of the Stueckelberg mass mixing, for the case when only one mode is massless, there are no millicharged particles coupled to the photon field. Thus milli-charge couplings appear in this case only when the Stueckelberg mixing parameter $\epsilon$ is introduced. For the case when only one mode is massless the kinetic mixing by itself does not allow for a milli charge.

Thus, the milli charge in Stueckelberg model is to be contrasted with the model where one has two massless modes (the photon and the paraphoton) [26] and the photon can couple with the hidden sector because of kinetic mixing generating milli-charged couplings. (An analysis with kinetic mixing and mass mixings of a different type is also considered in [27]).

\section{Electroweak Constraints}

Since the Standard Model is very successful in explaining physics at the electroweak scale, effects from new physics receive stringent constraints from electroweak observables. We concentrate our analysis on the StkSM where the effective coupling of new physics is

$\bar{\epsilon}=\frac{\epsilon-\delta}{\sqrt{1-\delta^{2}}}$.

As discussed earlier, StkSM and StSM are indistinguishable when considering electroweak constraints for the case where the hidden sector is absent. For the analysis of electroweak constraints, we do not consider the hidden sector matter.

The Stueckelberg mechanism predicts a mass shift for vector $Z$ boson. To be consistent with electroweak precision tests, such a shift must be small enough so that it lies within the error corridor of SM prediction.

\begin{tabular}{lrr}
\hline $\operatorname{Exp}(\Delta)$ & LEP Fit(Pull) & St Fit(Pull) \\
\hline$\Gamma_{Z}=2.4952(.0023)$ & $2.4956(-0.17)$ & $2.4956(-0.17)$ \\
$\sigma_{\mathrm{had}}=41.541(.037)$ & $41.476(1.76)$ & $41.469(1.95)$ \\
$R_{e}=20.804(.050)$ & $20.744(1.20)$ & $20.750(1.08)$ \\
$R_{\mu}=20.785(.033$ & $20.745(1.21)$ & $20.750(1.06)$ \\
$R_{\tau}=20.764(.045)$ & $20.792(-0.62)$ & $20.796(-0.71)$ \\
$R_{b}=0.21643(.00072)$ & $0.21583(0.83)$ & $0.21576(0.93)$ \\
$R_{c}=0.1686(.0047)$ & $0.17225(-0.78)$ & $0.17111(-0.53)$ \\
$A_{F B}^{(0, e)}=0.0145(.0025)$ & $0.01627(-0.71)$ & $0.01633(-0.73)$ \\
$A_{F B}^{(0, \mu)}=0.0169(.0013)$ & $0.01627(0.48)$ & $0.01633(0.44)$ \\
$A_{F B}^{(0, \tau)}=0.0188(.0017)$ & $0.01627(1.49)$ & $0.01633(1.45)$ \\
$A_{F B}^{(0, b)}=0.0991(.0016)$ & $0.10324(-2.59)$ & $0.10344(-2.71)$ \\
$A_{F B}^{(0, c)}=0.0708(.0035)$ & $0.07378(-0.85)$ & $0.07394(-0.90)$ \\
$A_{F B}^{(0, s)}=0.098(.011)$ & $0.10335(-0.49)$ & $0.10355(-0.50)$ \\
$A_{e}=0.1515(.0019)$ & $0.1473(2.21)$ & $0.1476(2.05)$ \\
$A_{\mu}=0.142(.015)$ & $0.1473(-0.35)$ & $0.1476(-0.37)$ \\
$A_{\tau}=0.143(.004)$ & $0.1473(-1.08)$ & $0.1476(-1.15)$ \\
$A_{b}=0.923(.020)$ & $0.93462(-0.58)$ & $0.93464(-0.58)$ \\
$A_{c}=0.671(.027)$ & $0.66798(0.11)$ & $0.66812(0.11)$ \\
$A_{s}=0.895(.091)$ & $0.93569(-0.45)$ & $0.93571(-0.45)$ \\
\hline & $\left(\chi^{2}=25.0\right)$ & $\left(\chi^{2}=25.2\right)$ \\
\hline
\end{tabular}

Table 1

Fits to $19 Z$ pole observables. The 1 st column is given by the PDG [28] with uncertainties $\Delta$. The 2nd column is from the SM Fit of the LEP EWWG [29]. The 3 rd column is an analysis for one StkSM model with inputs $\epsilon=0.06, \delta=0.03$, and $M_{1}=200 \mathrm{GeV}$. The Pull is defined as $(\operatorname{Exp}-$ Fit $) / \Delta$, and $\chi^{2}=\sum \mathrm{Pull}^{2}$. Taken from [24].

In the limit of $M_{1} \gg M_{Z}$, one finds an approximate upper bound on the mixing parameter $\bar{\epsilon}$ [30]

$|\bar{\epsilon}| \leq 0.061 \sqrt{1-\left(M_{Z} / M_{1}\right)^{2}}$.

Another constraint comes from the LEPII experiments where the new physics is typically characterized by the contact interaction parameter $\Lambda$. Among different contact interactions analyzed by LEPII group, one finds that the $\Lambda_{V V}>(21.7,17.1)$ $\mathrm{TeV}$ [31] usually gives the most stringent bound on $\bar{\epsilon}$. The StkSM predicts the theoretical value of $\Lambda_{V V}$ through the following formula

$$
\Lambda_{V V}=\frac{M_{Z^{\prime}}}{M_{Z}} \sqrt{\frac{4 \pi}{\sqrt{2} G_{F} v_{e}^{\prime 2}}} .
$$




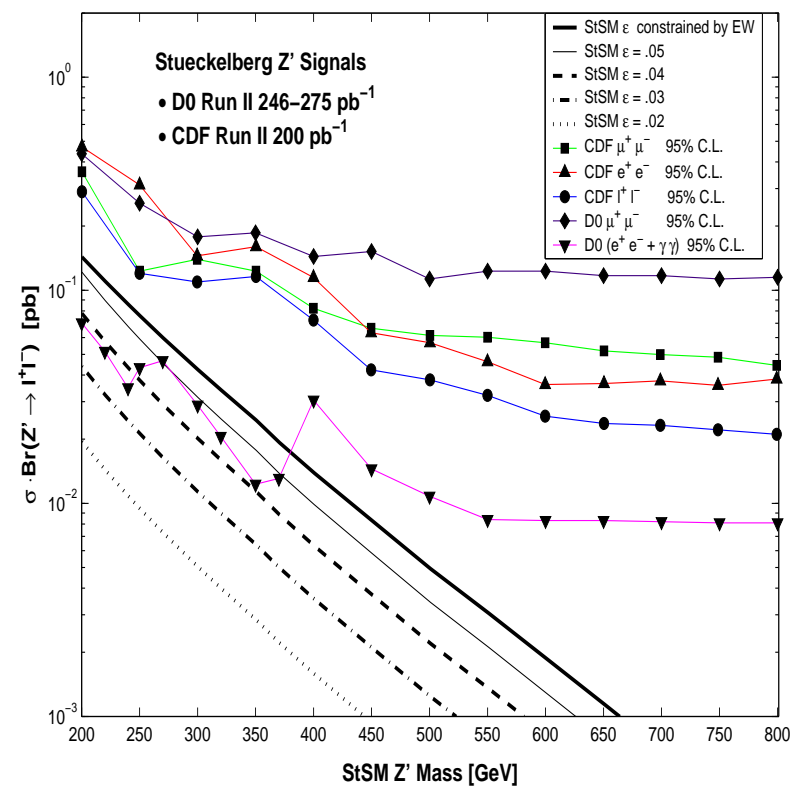

Figure 1. An exhibition of the $Z^{\prime}$ leptonic signal for different $\epsilon$ values in StSM, in comparison with the CDF [32] data with $200 \mathrm{pb}^{-1}$ and D0 [33] data with $246-275 \mathrm{pb}^{-1}$. The data (at $95 \%$ C.L.) puts a lower limit of about $250 \mathrm{GeV}$ on $M_{Z^{\prime}}$ for $\epsilon \approx 0.035$ and $375 \mathrm{GeV}$ for $\epsilon \approx 0.06$. Figure taken from [30].

However, even more stringent constraints come from fits to the high precision LEP data on the branching ratios of the $Z$ decay and the various asymmetries at the $Z$ pole, when one demands that the $\chi^{2}$ of the StkSM fits lie within $1 \%$ of that of the Standard Model. Table.(1) gives one example of the global fits to 19 observables for one specific StkSM model in the parameter space with $\bar{\epsilon} \sim 0.03$ and $M_{Z^{\prime}} \sim 200 \mathrm{GeV}$. As exhibited in Table.(1), the StkSM fits the 19 electroweak observables as well as the Standard Model does. As we will see in the next section, at $200 \mathrm{GeV}$ scale, a $Z^{\prime}$ signal controlled by the effective coupling $\bar{\epsilon}$ can be probed at the Tevatron collider with current and near future integrated luminosity. For a $Z^{\prime}$ resonance at a higher scale, one expects the constraints from the electroweak observable fits to become more relaxed than that at the scale close to the $Z$ scale.

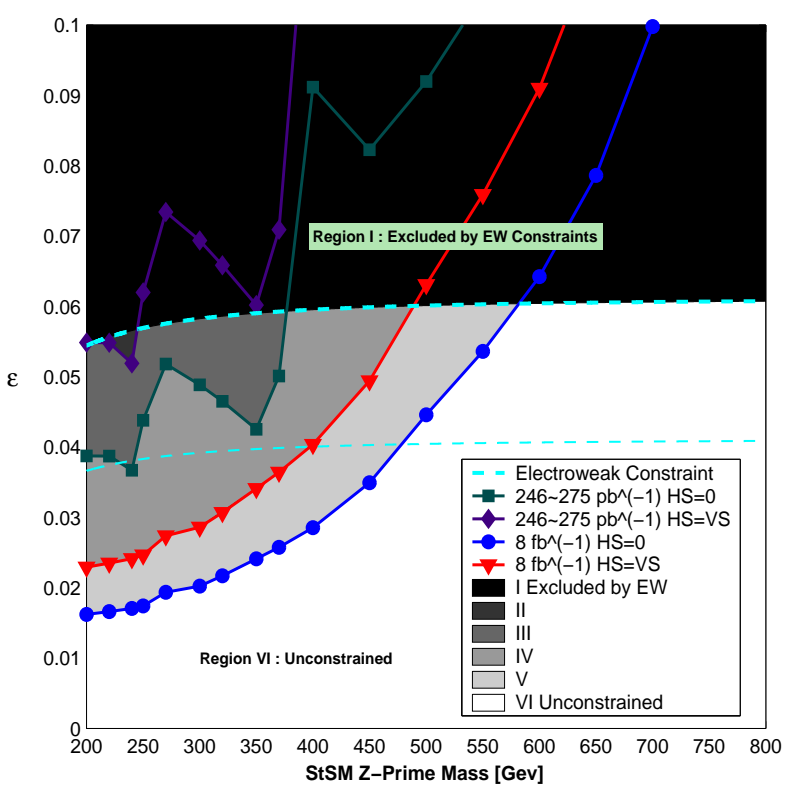

Figure 2. Exclusion plots in the $\epsilon-M_{Z^{\prime}}$ plane utilizing the more sensitive D0 [33] $e^{+} e^{-}+\gamma \gamma$ mode with (a) the $246-275 \mathrm{pb}^{-1}$ of data, and (b) $8 \mathrm{fb}^{-1}$ of data where an extrapolation of the sensitivity curve is used. Cases with a naive inclusion of hidden sector decay are also shown. Regions II, III, IV, and V are constrained by the conditions given at their respective boundaries. Figure taken from [30].

\section{Probing Narrow $Z^{\prime}$ Resonance at Tevatron}

One of the most promising channels to discover a new resonance at hadron colliders is the clean leptonic final states in the Drell-Yan process. In StSM, we are more interested in the energy region where two partons fuse into a $Z^{\prime}$ boson and then the $Z^{\prime}$ boson decays into dileptons.

A detailed analysis of the Drell-Yan cross section for the process $p \bar{p} \rightarrow Z^{\prime} \rightarrow \ell^{+} \ell^{-}$as a function of $M_{Z^{\prime}}$ in StSM is given in Fig.(1). The analysis is done at $\sqrt{s}=1.96 \mathrm{TeV}$, with a flat $K$ factor of 1.3 for the appropriate comparisons with other models and with the CDF [32] and D0 [33] combined data in the dilepton channel. (For more recent CDF data, see [34].) We integrate the dileptonic differential cross 


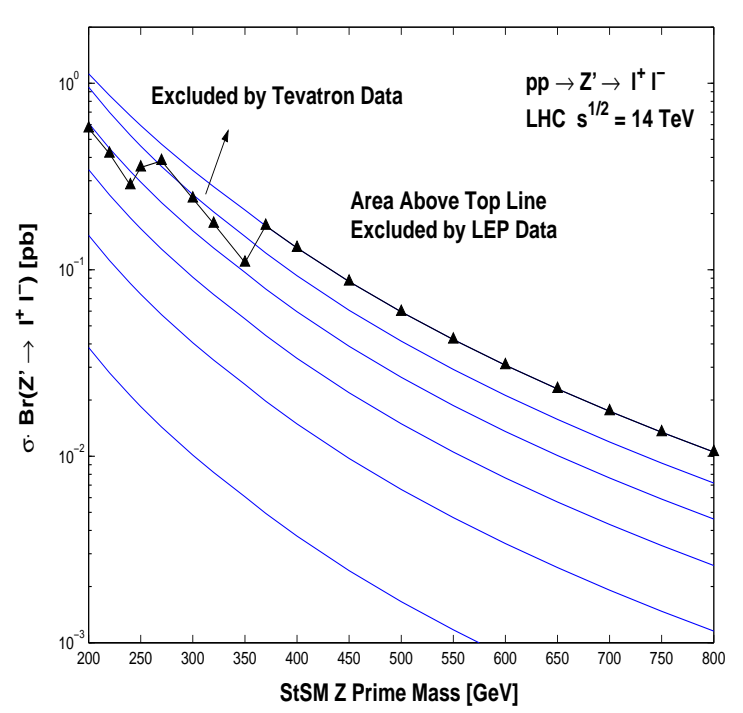

Figure 3. The prediction of the production cross section $\sigma\left(p p \rightarrow Z^{\prime}\right) \cdot \operatorname{Br}\left(Z^{\prime} \rightarrow \ell^{+} \ell^{-}\right)[\mathrm{pb}]$ for different $Z^{\prime}$ masses in the StSM at the LHC with the inclusion of the Tevatron constraints. The curves in descending order correspond to values of $\epsilon$ from .06 to .01 in steps of .01. Figure taken from [35].

section near the $Z^{\prime}$ resonance, and compare it with the experimental 95\% C.L. limits. Combining the theoretic calculation of the $Z^{\prime}$ resonance and the Tevatron data, one finds that the Stueckelberg $Z^{\prime}$ for the case $\epsilon \approx 0.06$ is eliminated up to about $375 \mathrm{GeV}$ (at $95 \%$ C.L.). This lower limit decreases as $\epsilon$ decreases but the data still constrains the model up to $\epsilon \approx 0.035$.

In Fig. (2) we give the exclusion plots in the $\epsilon-M_{Z^{\prime}}$ plane using the 246-275 $\mathrm{pb}^{-1}$ of data from D0 [33] and also using the total integrated luminosity of 8 $\mathrm{fb}^{-1}$ expected at the Tevatron. An analysis including the hidden sector with $\Gamma_{\mathrm{HS}}=\Gamma_{\mathrm{VS}}$ is also exhibited. The exclusion plots show that even the hidden sector is beginning to be constrained and these constraints will become even more severe with future data. The inclusion of the hidden sector here is only for illustrative purposes. A formal treatment of the hidden sector matter will be discussed later in the context of dark matter and LHC signatures.

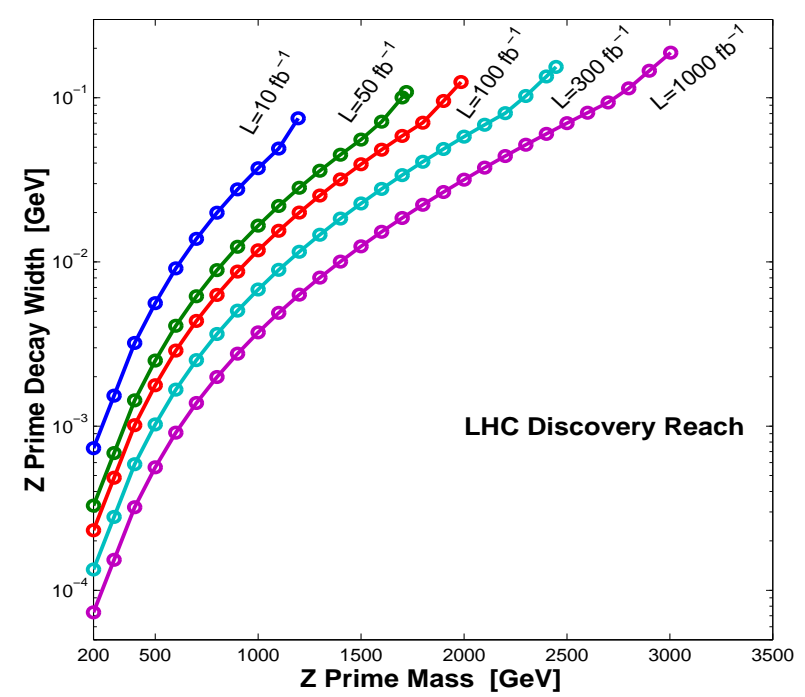

Figure 4. An analysis of the discovery reach of the LHC for StSM $Z^{\prime}$ decay widths and $Z^{\prime}$ masses. The regions that can be probed at LHC for a given luminosity are to the left and above each curve. Figure taken from [35].

\section{LHC Signatures of Narrow $Z^{\prime}$ Resonance}

Here we give an analysis for the exploration of the $Z^{\prime}$ boson at the LHC. The models considered here only consist of the case when the hidden sector fermions are absent or are kinematically inaccessible. If the $Z^{\prime}$ boson can decay into the hidden sector matter, the invisible decay widths usually dominate the visible widths, and thus dilute the LHC signatures. We discuss this further in details in the context of dark matter in the next section.

At the LHC, the promising signature channel for searching the $Z^{\prime}$ resonance is also the di-lepton final state, $p p \rightarrow Z^{\prime} \rightarrow \ell^{+} \ell^{-}$. The analysis of Fig.(3) predicts the dilepton signal $\sigma\left(p p \rightarrow Z^{\prime}\right) \cdot \operatorname{Br}\left(Z^{\prime} \rightarrow\right.$ $\left.\ell^{+} \ell^{-}\right)$in StSM that can be detected at LHC. Also plotted here is the signature region that has already been probed by Tevatron data in the mass regions up to $800 \mathrm{GeV}$.

Further we investigate the capability of the LHC in probing the mass and decay width of the Stueckel- 


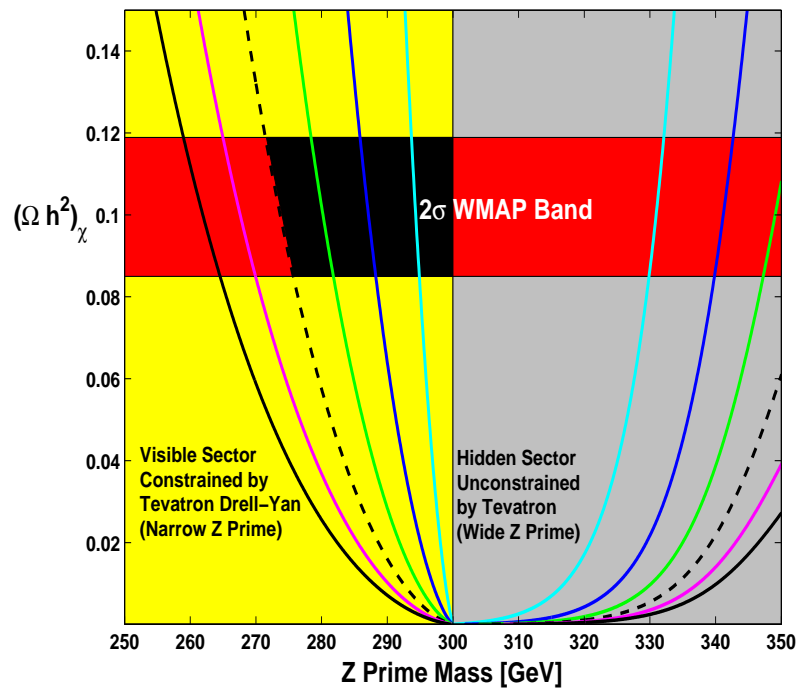

Figure 5. An analysis of the relic density of millicharged particles arising in the StSM from the hidden sector for the case $M_{\chi}=150 \mathrm{GeV}, \epsilon=(.01$ $.06)$ with .01 for the innermost curve and moving outward in steps of .01. The (yellow, grey) regions $\left(M_{Z^{\prime}}<2 M_{\chi}, M_{Z^{\prime}}>2 M_{\chi}\right.$ ) correspond to a (narrow, broad) $Z^{\prime}$ resonance, and the WMAP 3-year relic density constraints are satisfied for both cases. Figure taken from [24].

berg $Z^{\prime}$ boson. The criteria for the discovery of new physics is taken as $S>\operatorname{Max}(5 \sqrt{\mathrm{SM}}, 10)$. In Fig.(4) we give the discovery reach for finding the StSM $Z^{\prime}$ for different decay widths as a function of the $Z^{\prime}$ mass for luminosity in the range $10 \mathrm{fb}^{-1}$ and $1000 \mathrm{fb}^{-1}$. In the analysis we have assumed that detector effects can lead to signal and background losses of 50 percent. Here one finds that the LHC can probe a $100 \mathrm{MeV} Z^{\prime}$ up to about $2.75 \mathrm{TeV}$ and a $10 \mathrm{MeV}$ width up to a $Z^{\prime}$ mass of about $1.5 \mathrm{TeV}$.

\section{Milli Charge Dark Matter}

The milli-charged hidden sector matter in the Stueckelberg model is a natural candidate for the dark matter in the universe. Consider the model in which the hidden sector matter is a Dirac fermion $\chi$ which

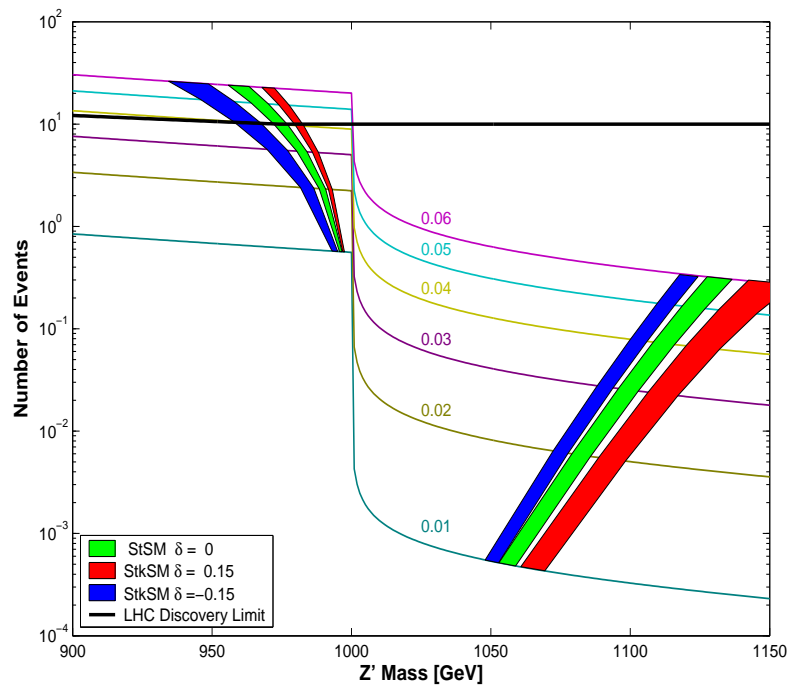

Figure 6. An exhibition of the dilepton signal as given by the number of events for $10 \mathrm{fb}^{-1}$ of integrated luminosity at the LHC consistent with the 3 year WMAP [38] relic density constraint as a function of $M_{Z^{\prime}}$ when $M_{\chi}=500 \mathrm{GeV}$. The curves in ascending order are for values of $\bar{\epsilon}$ in the range $(0.01-0.06)$ in steps of 0.01. Figure taken from [24].

couples to $U(1)_{X}$ field via [36]37|24]

$\mathcal{L}_{\text {int }}=g_{X} Q_{X} \bar{\chi} \gamma^{\mu} \chi C_{\mu}$

where $Q_{X}$ is the quantum number. Thus, the interactions between the hidden Dirac fermions and Standard Model particles are connected by vector bosons through the weak coupling parameter $\bar{\epsilon}$

$\chi \bar{\chi} \leftrightarrow A_{\gamma} / Z / Z^{\prime} \leftrightarrow f \bar{f}$.

Because the Dirac fermion $\chi$ has suppressed coupling to $A_{\gamma}$ and $Z$, and because $Z^{\prime}$ has suppressed coupling to the Standard Model fermions, $\chi$ will always couple weakly to Standard Model fermions. Thus it usually requires the Dirac fermion to annihilate near the vicinity of the $Z$ or $Z^{\prime}$ pole to be consistent with the relic abundance measured in WMAP experiments.

We discuss here only the annihilation near the $Z^{\prime}$ resonance. To compute the relic density of the universe, one has to properly integrate the annihilation 
cross section for various $\chi$ particles with different kinetic energy, and then obtain the thermally averaged annihilation rate $\langle\sigma v\rangle$. The thermal average near a resonance can produce significant deviations from naive estimates for the calculation of the dark matter relic density.

An example of the relic density analysis in StSM where $M_{\chi}=150 \mathrm{GeV}, \epsilon=(.01-.06)$ is exhibited in Fig.(5). It is shown that the relic density constraints from the 3 year WMAP data [38] can be satisfied over a broad range of the parameter space on both sides of the $Z^{\prime}$ pole.

As discussed earlier, one can discriminate the StkSM from StSM via the hidden sector interaction. We illustrate this effect in Fig.(6) along with LHC signatures. One finds that different bands that satisfy the relic density with different kinetic mixing parameter $\delta$ occupy different regions in the LHC signature space (the widths of the bands are translated from the error corridor of the relic density).

Another remarkable phenomenon from the hidden sector is its influence on the discovery reach of the $Z^{\prime}$ resonance at LHC. When $2 M_{\chi}<M_{Z^{\prime}}, Z^{\prime}$ can decay into hidden sector matter. The hidden sector decay width usually dominates the visible sector decay widths due to the normal coupling strength between $\chi$ and $Z^{\prime}$. In this case, the $Z^{\prime}$ produced at LHC will decay predominantly into hidden sector, and thus the hidden sector decay dilutes the visible leptonic signals at LHC as shown in Fig.(6). We note in passing that the searches of milli charged massive particles at the LHC are extremely difficult, and thus rely on sophisticated search strategies (for instance, see [39]). When $2 M_{\chi}>M_{Z^{\prime}}$, the hidden sector decay is turned off, the dileptonic signature is still the promising discovery channel at the LHC as discussed earlier.

\section{PAMELA Positron Excess and Breit-Wigner Enhancement}

The recent positron excess observed by PAMELA satellite [40] can be explained by dark matter annihilation in the galactic halo among other possible solutions. The positron excess requires the dark matter annihilation cross section in the galactic halo to be much larger than the annihilation cross section that gives rise to the relic density. This often leads to large boost factors whose physical origins are uncertain.

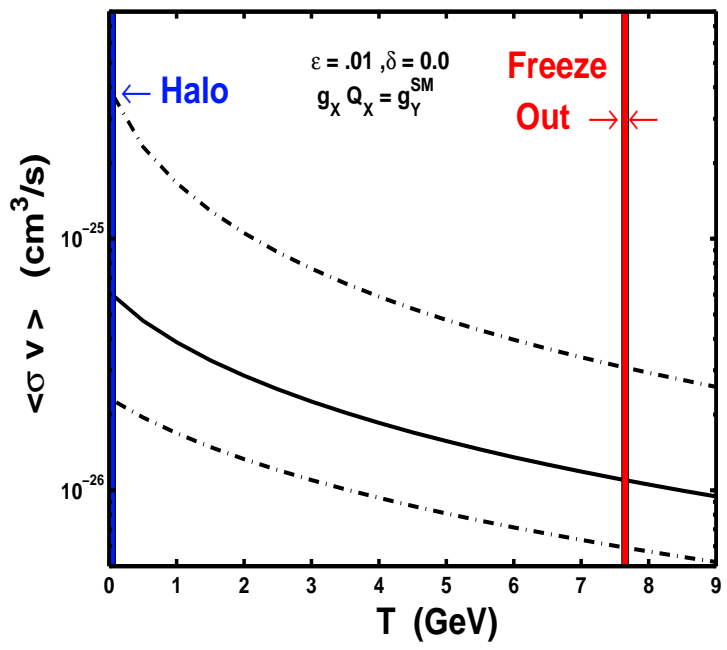

Figure 7. An exhibition of the dependence of $\langle\sigma v\rangle$ on temperature for Stueckelberg models with $M_{\chi}=$ $(150,151.5,153) \mathrm{GeV}$ and $M_{Z^{\prime}}=298 \mathrm{GeV}$. The annihilation near a pole generates a significant enhancement of $\langle\sigma v\rangle_{H}$ in the halo relative to $\left\langle\sigma v X_{f}\right.$ at the freezeout. The natural Breit-Wigner enhancement of $\langle\sigma v\rangle_{H}$ obviates the necessity of using very large boost factors. Figure taken from [41].

However, this difficulty can be overcome with annihilations near a $Z^{\prime}$ pole which is a narrow resonance in Stueckelberg models [41].

An illustration of the enhancement near the BreitWigner $Z^{\prime}$ pole is given in Fig.(7). The Breit-Wigner enhancement [41/42 43] can be achieved for the case when $M_{Z^{\prime}}<2 M_{\chi}$. For this case, the dark matter annihilates on the right hand side of the $Z^{\prime}$ pole. Thus, the $\langle\sigma v\rangle_{H}$ in the halo which annihilates near the resonance is much larger than $\langle\sigma v\rangle X_{f}$ at the freezeout in the early universe which annihilates far away from the resonance. The mass relation also implies that $Z^{\prime}$ is a narrow resonance which is essential for producing sufficient amounts of enhancement.

A detailed analysis of the positron excess in StSM is given in Fig.(8) where $M_{Z^{\prime}}=298 \mathrm{GeV}, M_{\chi}=150$ $\mathrm{GeV}$, and $\epsilon=0.006$. It is shown that the BreitWigner enhancement near the $Z^{\prime}$ pole can produce 


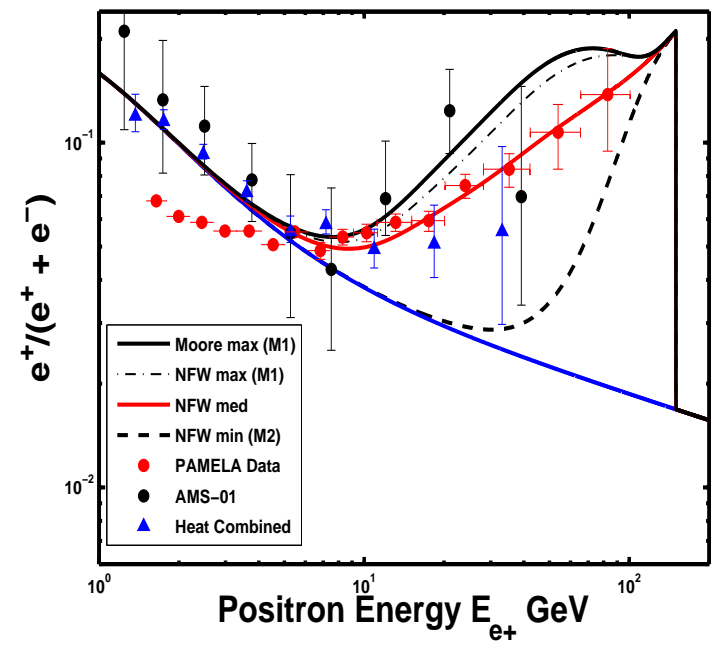

Figure 8. Positron spectrum including the monochromatic source and continuum flux in StSM with model inputs $\epsilon=0.006, M_{Z^{\prime}}=298 \mathrm{GeV}$ and $M_{\chi}=150$ $\mathrm{GeV}$, for various halo profiles and diffusion models. Also plotted here is the PAMELA data [40], along with the AMS-01 and HEAT data [44]. The background flux ratio is the decaying solid (blue) lower curve. Figure taken from [41].

the positron spectrum in the energy range $10 \sim 100$ $\mathrm{GeV}$ observed by PAMELA. We note in passing that the Stueckelberg $Z^{\prime}$ has larger lepton branching ratios than the SM $Z$ and other SM like $Z^{\prime}$ because of the mixing between $U(1)_{X}$ and hypercharge $U(1)_{Y}$. Thus the $Z^{\prime}$ does not produce too much hadronic final states. (For a recent review of different heavy $Z^{\prime}$ models, see [45].) The parameter region $M_{Z^{\prime}}<2 M_{\chi}$ that is favored by the PAMELA data is also the region that has promising LHC signatures as discussed before.

\section{Conclusion}

The Stueckelberg mechanism allows for an Abelian gauge boson mass growth without the benefit of a Higgs mechanism. In the Stueckelberg extension of the Standard Model (StSM), the Stueckelberg Lagrangian enlarges the neutral vector boson sector which then leads to modifications of the $Z$ boson mass, the electric charge, and the hypercharge coupling constant. In the Stueckelberg extension of the Standard Model with kinetic mixing (StkSM) but without hidden sector matter, the kinetic mixing gets absorbed by the Stueckelberg mass mixing, which renders StkSM indistinguishable from StSM in the SM electroweak sector. A discrimination of the StkSM from StSM comes about due to the presence of hidden sector matter. Further, if matter exists in the hidden sector, it must be milli charged and here it is interesting to note that the milli charge of the hidden sector matter is generated by the Stueckelberg mass mixing parameter, and not the kinetic mixing parameter.

The new physics arising in Stueckelberg models is controlled by the Stueckelberg mass mixing parameter which must be small to be consistent with the electroweak precision test. However this small mixing parameter can still lead to detectable $Z^{\prime}$ signals which can manifest as a narrow resonance at the Tevatron and at the LHC. Tevatron searches from D0 and CDF have begun to probe the parameter space of Stueckelberg models in the mass region where many other $Z^{\prime}$ s have been excluded. The LHC prediction of the leptonic signals of the Stueckelberg $Z^{\prime}$ shows that one can detect this narrow resonance in a broad range of $Z^{\prime}$ mass.

The milli-charged dark matter in the hidden sector couples with $Z^{\prime}$ with normal strength, so the $Z^{\prime}$ will decay into the hidden sector predominantly if allowed, and for that case the $Z^{\prime}$ signal is diluted completely at LHC. Due to the weak coupling strength between the milli-charged dark matter and the Standard Model particles, the milli-charged dark matter has to annihilate in the vicinity of the $Z$ or $Z^{\prime}$ pole to produce the right amount of dark matter to be consistent with the relic abundance measured by WMAP. The PAMELA positron excess anomaly can be naturally explained by the Breit-Wigner enhancement near the $Z^{\prime}$ resonance in Stueckelberg models. The Breit-Wigner enhancement occurs in the same region of the parameter space where the $Z^{\prime}$ is a narrow resonance and the leptonic signature is strong, and thus detectable at the LHC.

Acknowledgments: This research was supported in part by NSF grant PHY-0653342. 


\section{REFERENCES}

1. E. C. G. Stueckelberg, Helv. Phys. Acta.11, 225 (1938).

2. V. Ogievetskii, and I. V. Polubarinov, JETP 14, 179 (1962).

3. M. Kalb, and P. Ramond, Phys. Rev. D9, 2273 (1974).

4. F. Cianfrani and O. M. Lecian, Nuovo Cim. 122B, 123 (2007) [arXiv:physics/0701340].

5. F. Cianfrani and O. M. Lecian, Int. J. Mod. Phys. A 23, 1105 (2008) [arXiv:0803.1583 [physics.hist-ph]].

6. B. Kors and P. Nath, JHEP 0412, 005 (2004) [arXiv:hep-ph/0406167].

7. B. Kors and P. Nath, arXiv:hep-ph/0411406

8. B. Kors and P. Nath, JHEP 0507, 069 (2005) [arXiv:hep-ph/0503208].

9. D. Feldman, B. Kors and P. Nath, Phys. Rev. D 75, 023503 (2007) [arXiv:hep-ph/0610133].

10. D. Feldman, Z. Liu and P. Nath, AIP Conf. Proc. 939, 50 (2007) [arXiv:0705.2924 [hep-ph]].

11. D. Feldman, Z. Liu and P. Nath, AIP Conf. Proc. 1078, 116 (2009) [arXiv:0806.4683 [hep-ph]].

12. P. Nath, arXiv:0812.0958 [hep-ph].

13. D. Feldman, Z. Liu, P. Nath and B. D. Nelson, arXiv:0907.5392 [hep-ph].

14. P. Anastasopoulos, Fortsch. Phys. 55, 633 (2007).

15. J. Kumar, A. Rajaraman and J. D. Wells, Phys. Rev. D 77, 066011 (2008).

16. P. Anastasopoulos, F. Fucito, A. Lionetto, G. Pradisi, A. Racioppi and Y. S. Stanev, Phys. Rev. D 78, 085014 (2008).

17. J. De Rydt, T. T. Schmidt, M. Trigiante, A. Van Proeyen and M. Zagermann, JHEP 0812, 105 (2008).

18. R. Armillis, C. Coriano', M. Guzzi and S. Morelli, Nucl. Phys. B 814, 15679 (2009).

19. C. Coriano, M. Guzzi, N. Irges and A. Mariano, Phys. Lett. B 671, 87 (2009).

20. C. Coriano, M. Guzzi, A. Mariano and S. Morelli, Phys. Rev. D 80, 035006 (2009).

21. Y. Zhang, S. Z. Wang and Q. Wang, JHEP 0803, 047 (2008).

22. Y. Mambrini, arXiv:0907.2918 [hep-ph].

23. B. Kors and P. Nath, Phys. Lett. B 586, 366 (2004) [arXiv:hep-ph/0402047].

24. D. Feldman, Z. Liu and P. Nath, Phys. Rev. D 75,
115001 (2007) [arXiv:hep-ph/0702123].

25. S. A. Abel, M. D. Goodsell, J. Jaeckel, V. V. Khoze and A. Ringwald, JHEP 0807, 124 (2008) [arXiv:0803.1449 [hep-ph]].

26. B. Holdom, Phys. Lett. B 166, 196 (1986).

27. B. Holdom, Phys. Lett. B 259, 329 (1991).

28. S. Eidelman et al. [Particle Data Group], Phys. Lett. B 592, 1 (2004).

29. [ALEPH Collaboration and DELPHI Collaboration and L3 Collaboration and ], Phys. Rept. 427, 257 (2006).

30. D. Feldman, Z. Liu and P. Nath, Phys. Rev. Lett. 97, 021801 (2006) [arXiv:hep-ph/0603039].

31. [LEP Collaboration], arXiv:hep-ex/0312023; S. Schael et al. [ALEPH Collaboration], Eur. Phys. J. C 49, 411 (2007).

32. A. Abulencia et al. [CDF Collaboration], Phys. Rev. Lett. 95, 252001 (2005).

33. V. M. Abazov et al. [D0 Collaboration], Phys. Rev. Lett. 95, 091801 (2005).

34. T. Aaltonen et al. [CDF Collaboration], Phys. Rev. Lett. 102, 031801 (2009).

35. D. Feldman, Z. Liu and P. Nath, JHEP 0611, 007 (2006) [arXiv:hep-ph/0606294].

36. K. Cheung and T. C. Yuan, JHEP 0703, 120 (2007) |arXiv:hep-ph/0701107|.

37. K. m. Cheung and T. C. Yuan, arXiv:0710.2005 [hep-ph].

38. D. N. Spergel et al. [WMAP Collaboration], Astrophys. J. Suppl. 170, 377 (2007).

39. J. Chen and T. Adams, arXiv:0909.3157 [hep$\mathrm{ph}$.

40. O. Adriani et al. [PAMELA Collaboration], Nature 458, 607 (2009).

41. D. Feldman, Z. Liu and P. Nath, Phys. Rev. D 79, 063509 (2009) [arXiv:0810.5762 [hep-ph]].

42. M. Ibe, H. Murayama and T. T. Yanagida, Phys. Rev. D 79, 095009 (2009) arXiv:0812.0072 [hep-ph]].

43. W. L. Guo and Y. L. Wu, Phys. Rev. D 79, 055012 (2009) [arXiv:0901.1450 [hep-ph]].

44. S. W. Barwick et al. [HEAT Collaboration], Astrophys. J. 482, L191 (1997); M. Aguilar et al. [AMS-01 Collaboration], Phys. Lett. B 646 (2007) 145.

45. P. Langacker, Rev. Mod. Phys. 81, 1199 (2009) [arXiv:0801.1345 [hep-ph]]. 